\title{
Screening of Paroxysmal Atrial Fibrillation after Ischemic Stroke: 48-Hour Holter Monitoring versus Prolonged Intermittent ECG Recording
}

\author{
Gustav Orrsjö, ${ }^{1,2}$ Björn Cederin, ${ }^{1}$ Eric Bertholds, ${ }^{1}$ Salmir Nasic, ${ }^{2}$ and Lennart Welin ${ }^{3,4}$ \\ ${ }^{1}$ Department of Internal Medicine, Skaraborgs Sjukhus Skövde, 54152 Skövde, Sweden \\ ${ }^{2}$ Department of Research and Development, Skaraborgs Sjukhus Skövde, 54152 Skövde, Sweden \\ ${ }^{3}$ Department of Internal Medicine, Skaraborgs Sjukhus Lidköping, 53185 Lidköping, Sweden \\ ${ }^{4}$ University of Gothenburg, P.O. Box 100, 40530 Gothenburg, Sweden
}

Correspondence should be addressed to Gustav Orrsjö; gustav.orrsjo@vgregion.se

Received 6 December 2013; Accepted 9 January 2014; Published 4 March 2014

Academic Editors: E. Berge and M. Paciaroni

Copyright (C) 2014 Gustav Orrsjö et al. This is an open access article distributed under the Creative Commons Attribution License, which permits unrestricted use, distribution, and reproduction in any medium, provided the original work is properly cited.

\begin{abstract}
Aims. Screening of paroxysmal atrial fibrillation (PAF) after ischemic stroke and TIA is important. The ideal method is not known and studies of intermittent screening methods in particular are lacking. In this retrospective study we compared a shorter continuous screening method with an intermittent screening method. Methods. Since early 2011 our stroke unit has used two different methods of screening: either a 48-hour continuous screening with Holter monitor or a 21-day twice daily intermittent screening with a hand-held ECG recorder. Through the Swedish National Stroke Registry and medical records reviewing all screening episodes between 2011-02-01 and 2013-01-31 were collected and analysed. Results. Of 386 screenings, 26 screenings were excluded leaving 360 screenings for the final analysis of which 114 screenings were made with hand-held ECG recorder and 246 with Holter monitoring. No significant difference between the groups concerning basic characteristics was observed. In the hand-held ECG recorder group a total of 13 PAF screenings $(11.4 \%)$ were detected compared with $7(2,8 \%)$ in the Holter group $(P=0.001)$. Conclusions. A prolonged intermittent screening is a better method than a shorter continuous screening in terms of detecting PAF after ischemic stroke and TIA.
\end{abstract}

\section{Introduction}

Stroke is the third most common cause of death in most Western populations after coronary heart disease and cancer. The majority of these strokes are ischemic strokes for which atrial fibrillation (AF) is a major risk factor, conferring a fivefold increased risk [1]. This increased risk appears to be the same regardless of whether the atrial fibrillation is permanent or paroxysmal $[2,3]$. Importantly, a diagnosis of atrial fibrillation after stroke usually results in secondary prophylaxis with oral anticoagulants, a highly effective treatment with a relative risk reduction of recurrence of $65 \%$ compared with placebo [4]. Furthermore, it has been suggested that paroxysmal atrial fibrillation (PAF) may be an underestimated cause of ischemic stroke [5, 6]. With the high efficacy of oral anticoagulants in mind, it is crucial to identify all significant PAF in order to achieve the best possible secondary prevention. Since PAF often is asymptomatic, it requires screening to be diagnosed [7]. In addition, screening for PAF after ischemic stroke was in a recent model shown to be cost effective [8].

Up until now in-patient continuous cardiac monitoring has been the most common method to identify PAF, usually for the first 24 or 48 hours after admission. The longer the screening is done, the better the yield is in terms of detected PAF $[9,10]$. However, a long term continuous Holter screening strategy may be associated with lower compliance and technical problems [10, 11]; furthermore the amount of data to be analyzed increases. If other methods such as implantable loop recorders or pacemakers are considered, their potential downsides are cost and invasiveness [11]. With this in mind an intermittent screening is an attractive 

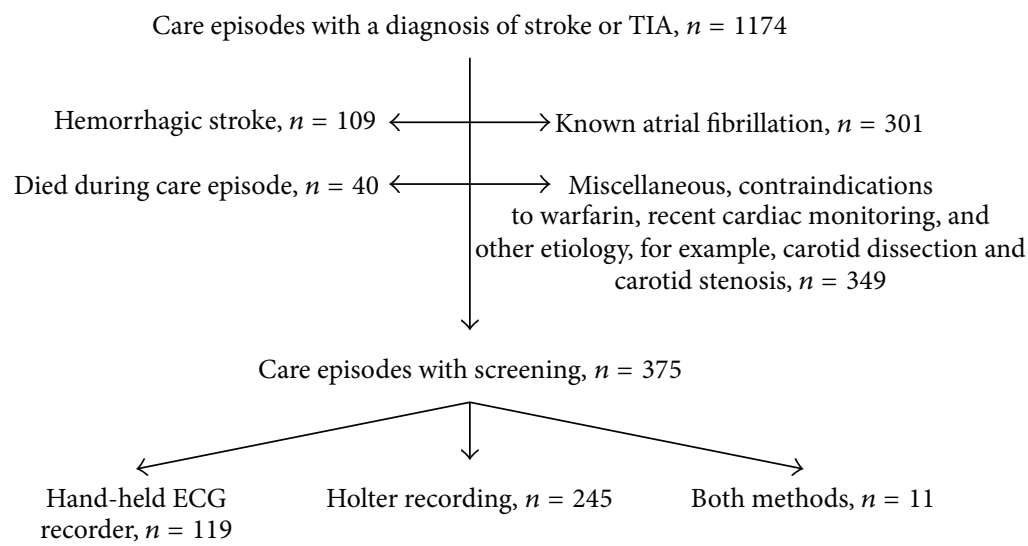

FIgURE 1: Total number of screenings, $n=386(119+245+2 \times 11)$.

alternative method and recent studies have shown promising results $[12,13]$. However, clinical data of efficacy of and feasibility in a routine clinical setting is lacking.

In our stroke ward we have used two different methods: a 48-hour Holter method and since early 2011 an intermittent long term screening method with a novel patient triggered event recorder. The aim of this retrospective study was to compare these two methods regarding efficacy and feasibility in a routine clinical setting of unselected stroke and TIA patients.

\section{Material and Methods}

The study population was retrospectively selected from the Swedish National Stroke Registry where all care episodes with TIA or stroke diagnoses are collected [14]. From this registry we selected all the hospital care episodes in the municipality of east Skaraborg during the decided time frame from the 1st of February 2011 to the 31st of January 2013, in total 1174 inhospital care episodes. Of these 1174 care episodes screening for PAF was made in 375 cases. The decision whether to screen or not was made by the clinician and based on if the patient was considered suitable for anticoagulant treatment. Through medical records the reasons not to screen were obtained and are shown in Figure 1.

Two different methods of screening were used: either a continuous 48-hour monitoring strategy with routine Holter monitors (DL 700/10, Braemar Inc.) or an intermittent strategy with a novel hand-held ECG recorder. The handheld ECG recorder (ZenicorEKG-2; Zenicor Medical Systems $\mathrm{AB}$ ) is a small rectangular box with two sensors and a display in the center. When applying the thumbs on the two sensors for 30 seconds a bipolar ECG corresponding lead 1 is created. Through mobile phone technology the ECG is transferred to a secure database from where it can be analyzed. The method has earlier been shown feasible for screening with high sensitivity and specificity with respect to diagnosing atrial fibrillation [15]. The method used was dependent on if hand-held ECG recorders were available at the ward preceding the start of the screening. If unavailable the patient was referred to the clinical physiology laboratory for a 48-hour Holter monitoring. Before the Holter monitor was hitched, a routine ECG was done. If atrial fibrillation was present on this ECG $(n=1)$, it was considered a positive screening as it was a part of the Holter method.

Patients receiving the hand-held ECG recorder were instructed to perform rhythm recording twice daily for three weeks (during 1 February-21 March 2011 for 30 days) in addition to when experiencing arrhythmia symptoms, with each recording lasting 30 seconds. After the three weeks the patient was told to leave the device at the local primary care center that sent the device back to the ward, where a new patient was equipped.

The interpretations of the hand-held ECG recordings and Holter recordings were made by biomedical scientists at the clinical physiology laboratory. The biomedical scientists were continuously reviewing the hand-held ECG recordings and when a positive episode was detected, the screening was interrupted. If in doubt when interpreting the recordings the biomedical scientists had access to cardiologists for support. Neither the biomedical scientists nor the cardiologists were part of our study group.

The screening was considered positive for each method when an episode of atrial fibrillation lasting for 30 seconds or longer was captured, which is in accordance with current practice [16]. Episodes lasting less than 30 seconds were considered a negative screening outcome and these patients received no anticoagulation.

Of the 375 care episodes that were screened, 11 care episodes utilized both screening methods; that is, the total number of screenings was 386. The reason for using both methods was due to quality problems $(n=7)$ and misunderstanding $(n=4)$. Medical records and former ECG recordings were then scrutinized and exclusion criteria were applied (Table 1). The results of the screenings were then obtained from reviewing medical records and the database where the intermittent hand-held ECG recordings were collected. Patient characteristics were collected from the Swedish National Stroke Registry and from a careful review of medical records. When patients included in the final analysis 
TABLE 1: Reasons for exclusion.

\begin{tabular}{lccc}
\hline Exclusion criteria & Holter $(n=256)$ & Hand-held $(n=130)$ & 3 \\
History of atrial fibrillation* & 8 & 5 & Total $(n=386)$ \\
Lack of patient compliance/misunderstanding $^{* *}$ & 1 & 5 & 6 \\
Technical problems/qualitative problems ${ }^{* *}$ & 1 & 3 & 6 \\
Premature screening interruption of other causes & 0 & $\mathbf{1 6}$ & 3 \\
Total screening episodes excluded & $\mathbf{1 0}$ & 114 & $\mathbf{2 6}$ \\
Screening episodes remaining & 246 & 360 \\
\hline
\end{tabular}

${ }^{*} 4 / 8$ in the Holter group and 3/3 in the hand-held ECG group were screened positive; ${ }^{* *}$ if less than 24 -hour recording, 12 hand-held ECG recordings were interpretable.

TABLE 2: Basic patient characteristics of screening (number of patients $=351$ ).

\begin{tabular}{|c|c|c|c|c|}
\hline Parameter & Hand-held ECG recorder & Holter & All & $P$ value \\
\hline Number of screenings & 114 & 246 & 360 & - \\
\hline Ischemic stroke patients (\%) & 72.8 & 67.9 & 69.4 & $0.35^{1}$ \\
\hline TIA patients (\%) & 27.2 & 32.1 & 30.6 & $0.35^{1}$ \\
\hline TACI $(\%) n=250$ & 2.4 & 1.8 & 2.0 & \\
\hline PACI $(\%) n=250$ & 20.5 & 19.2 & 19.6 & \\
\hline LACI $(\%) n=250$ & 48.2 & 46.1 & 46.8 & $0.94^{1}$ \\
\hline POCI $(\%) n=250$ & 21.7 & 22.8 & 22.4 & \\
\hline Unclassified (\%) & 7.2 & 10.2 & 9.2 & \\
\hline Age (years), mean (range) & $70.3(41.9-86.6)$ & $70.4(42.4-89.6)$ & $70.4(41.9-89.6)$ & $0.91^{2}$ \\
\hline Gender male (\%) & 67.5 & 58.1 & 61.1 & $0.09^{1}$ \\
\hline Known diabetes mellitus (\%) & 16.7 & 19.5 & 18.6 & $0.52^{1}$ \\
\hline Known hypertension (\%) & 58.8 & 56.1 & 56.9 & $0.63^{1}$ \\
\hline Previous stroke (\%) & 16.7 & 11.4 & 13.1 & $0.17^{1}$ \\
\hline Ischemic heart disease (\%) & 15.8 & 13.4 & 14.2 & $0.55^{1}$ \\
\hline Current smoking (\%) & 23.7 & 19.5 & 20.8 & $0.37^{1}$ \\
\hline NIHSS at admission, median (quartiles) $n=250$ & $2(1-3)$ & $2(1-4)$ & 2 & $0.17^{3}$ \\
\hline
\end{tabular}

${ }^{1}$ Statistical analysis made with Chi-square; ${ }^{2}$ statistical analysis made with Students $t$-test; ${ }^{3}$ statistical analysis made with Mann-Whitney $U$ test.

had two care episodes with screening $(n=5)$ or used both methods $(n=4)$, they were still considered as two different observations.

Statistical analysis was done using SPSS v.20. To compare the groups with respect to categorical variables a Pearson Chisquare test was performed, for normal distributed variables (age) a Student $t$-test was performed, and for the nonnormal distributed variables (NIHss) a Mann-Whitney test was performed. A $P$ value below 0.05 was considered statistically significant. The study was approved by the regional ethical review board in Gothenburg (registration number 580-13).

\section{Results}

Of a total of 386 screenings, 26 were excluded leaving 360 screening episodes to be analyzed (Table 1). Of the patients excluded due to a history of atrial fibrillation $(n=11), 4 / 8$ in the Holter group and 3/3 in the hand-held ECG recorder group were screened positive.

A total number of 360 screenings in 351 patients with recent stroke and TIA were analyzed, of which 114 were made with the hand-held ECG recorder and 246 with Holter monitor. Their basic characteristics are shown in Table 2. None of the basic characteristics differed significantly between the groups although a tendency towards more men in the handheld ECG recorder group could be observed $(P=0.09)$.

In the Holter group 7 (2,8\%) PAF screening were detected compared with $13(11,4 \%)$ in the hand-held ECG recorder group (Table 3 ). The difference was highly significant $(P=$ $0.001)$.

The mean starting point for the screening in the Holter group was 14.2 days (median 6, range 0-94) after stroke onset. The mean recording time was 46.7 hours (range $24-48$ hours) and the mean time of atrial fibrillation among the positive screenings was 12.3 hours (range 0,025-48 hours). In the hand-held ECG group the mean starting point was 4.1 days (median 2, range 0-38) after stroke onset. The total number of 30-second recordings was 5810, of which 955 (16.3\%) exceeded the twice daily instruction. The mean number of recording episodes was 51 (range $3-137$, median 48 ) and the mean time of screening duration was 22.2 days (range 1-48, median 21). The mean day of PAF detection after screening start was day number 8 (range 1-22) (Figure 2). The mean number of recording episodes with atrial fibrillation in each positive screening was 3.9 (range 1-11). When accumulating the time from the first to the last positive screening episodes 
TABLE 3: Detection of PAF with the two methods.

\begin{tabular}{lcccc}
\hline Method used & Positive screening $(n)$ & Negative screening $(n)$ & All screenings total $(n)$ & Detection rate $(\%)$ \\
\hline Hand-held ECG recorder $(n)$ & 13 & 101 & 114 & 11.4 \\
48-hour Holter monitoring $(n)$ & 7 & 239 & 246 & 2.8 \\
\hline
\end{tabular}

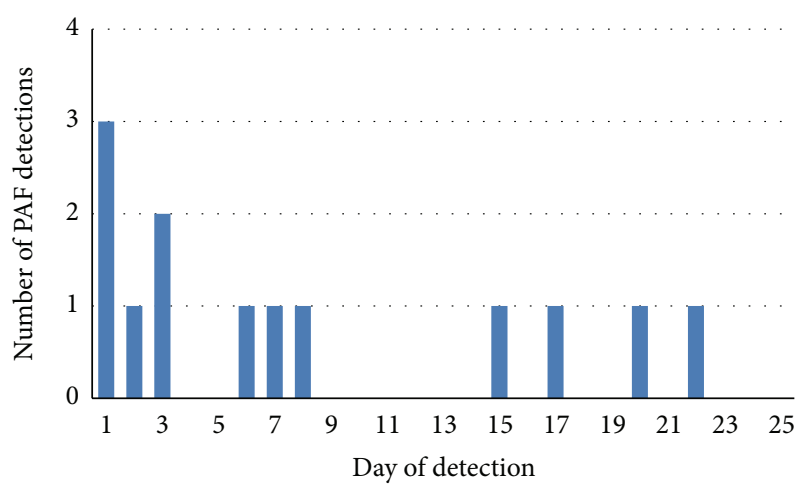

Figure 2: Day after screening start of PAF detection by hand-held ECG recorder.

among the patients with more than 1 positive screening episode $(n=10)$ the mean accumulated time of continuous positive recordings was 26 hours (Figure 3 ).

\section{Discussion}

In this retrospective study we have shown that intermittent long term screening yields more than short term continuous screening in terms of detecting paroxysmal atrial fibrillation among patients with recent stroke or TIA. A shorter continuous screening of 48 hours is not enough to exclude a potential PAF, missing 3/4 of those who would have been diagnosed with a longer intermittent screening. The novel hand-held ECG recorder was proven feasible for performing the intermittent recordings with low exclusion rates due to compliance and technical problems. This result accords with previous studies $[12,13]$ but has now been confirmed in a routine clinical setting.

The study has limitations. Firstly, the timing of the screening start differed between the groups, probably due to the referral related delay of Holter monitoring initiation. Our knowledge of how this affects the detection rate is scarce. In the EMBRACE study (presented at the 2013 International Stroke Conference, Honolulu, Hawaii, available at http://my .americanheart.org/idc/groups/ahamah-public/@wcm/@sop /@scon/documents/downloadable/ucm_449046.pdf) 80\% of the PAF: $s$ was detected during the first two weeks after screening start, and other studies have shown similar results [17]. Nevertheless, that the yield of the screening is most fruitful during the first two weeks is not equivalent to the assumption that the yield is dependent on timing of screening start after the index event. For instance, in the EMBRACE study the screening started about 70 days after stroke, would the yield be better or worse if it would have started 40 days after stroke? Secondly, in a retrospective

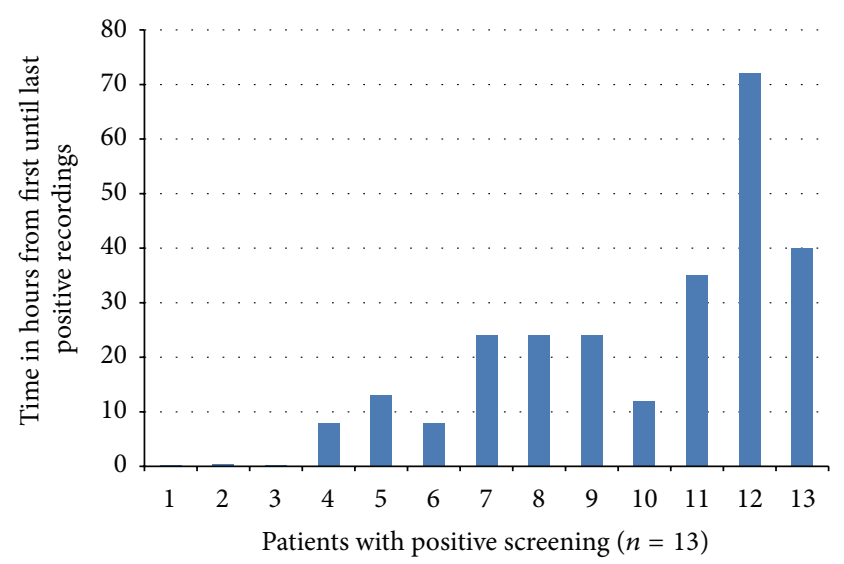

Figure 3: Accumulated time from first positive recording until last positive recording in all positive screenings.

study a potential selection bias is a limitation. One may argue that the clinician most probably took stroke severity and stroke characteristics into consideration when choosing screening method. However, that no significant difference could be found between the groups including NIHSS points and OCSP classification further supports the presumption that the screening methods were assigned depending on availability, limiting the probability of a selection bias. Thirdly, since epidemiological studies have shown that atrial fibrillation is more frequent in men, the observed tendency towards more male subjects in the hand-held group could have affected the final result [18]. Nevertheless, there is no evidence that gender predicts the detection of paroxysmal atrial fibrillation after stroke $[19,20]$.

The detection rate in other studies has been varied. In a review from 2011 the detection rate ranged from $1,0 \%$ to 13.9 in studies done with unselected stroke and TIA populations depending on the length of screening [11]. These figures imply that our figure of $11.3 \%$ in the long intermittent screening group may reflect a reasonable yield when choosing a longer screening. Our yield of $2.8 \%$ in the short term screening group was low compared with these figures and lower than expected. However, many of the patients in the Holter group had short episodes of supraventricular tachycardia, some of which were irregular with no certain p-waves, but all had duration of less than 30 seconds. In other studies the cutoffs have been set at five or ten seconds which might partially explain the difference in detection rate $[14,19]$.

The exclusion rate in both groups due to compliance and technical problems was low, although it was higher in the hand-held ECG group compared to the Holter group. In an intention to screen analysis the detection rate would drop to $10.5 \%$ in the hand-held ECG group but the difference 
between the groups would still be statistically significant. This finding stresses the feasibility of the hand-held ECG recorder in a clinical setting. Nevertheless, in a setting of more severe strokes, the dropout rates due to compliance problems might be higher. Among the patients excluded owing to a history of atrial fibrillation, the hand-held ECG recorder had a higher sensitivity of rediscovering PAF, which is in accordance with the overall results.

The mean duration of the intermittent screening exceeded the instructed 21 days, including all positive screenings prematurely interrupted. This can partly be explained by the 30-day screening instruction during the first seven weeks of the study period and partly by the fact that the patients keep the equipment longer than they are instructed to. Although most detections occurred during the first week (Figure 2), detections were made throughout the whole period. The ideal duration of screening is not known and this area, along with other questions of screening design, needs further research.

Finally we want to address the question of atrial fibrillation burden and absolute stroke risk, which is an area of ongoing debate with partially conflicting results in studies presented $[5,21,22]$. The exact amount of atrial fibrillation burden cannot be assessed with intermittent screening and we have no firm evidence of an increased stroke risk starting from 30 seconds of atrial fibrillation. However, when reflecting the fact that all positive screenings but three had more than one positive screening in a row (Figure 3), it seems that we are catching longer PAF episodes.

\section{Conclusion}

In conclusion, a longer intermittent screening strategy is preferable to a shorter continuous screening strategy. With its advantages, an intermittent screening with a hand-held ECG recorder is a tempting novel approach to increase PAF detection after TIA and ischemic stroke. Many questions about screening design, PAF detection predictors, and net clinical benefits of oral anticoagulant treatment in patients with screening detected PAF remain, which require future studies to be answered.

\section{Conflict of Interests}

The authors declare that there is no conflict of interests regarding the publication of this paper.

\section{References}

[1] P. A. Wolf, R. D. Abbott, and W. B. Kannel, "Atrial fibrillation as an independent risk factor for stroke: the Framingham study," Stroke, vol. 22, no. 8, pp. 983-988, 1991.

[2] S. H. Hohnloser, D. Pajitnev, J. Pogue et al., "Incidence of stroke in paroxysmal versus sustained atrial fibrillation in patients taking oral anticoagulation or combined antiplatelet therapy: an Active W substudy," Journal of the American College of Cardiology, vol. 50, no. 22, pp. 2156-2161, 2007.

[3] L. Friberg, N. Hammar, and M. Rosenqvist, "Stroke in paroxysmal atrial fibrillation: report from the Stockholm Cohort of
Atrial Fibrillation," European Heart Journal, vol. 31, no. 8, pp. 967-975, 2010

[4] G. W. Albers, D. G. Sherman, D. R. Gress, J. E. Paulseth, and P. Petersen, "Stroke prevention in nonvalvular atrial fibrillation: a review of prospective randomized trials," Annals of Neurology, vol. 30, no. 4, pp. 511-518, 1991.

[5] J. S. Healey, S. J. Connolly, M. R. Gold et al., "Subclinical atrial fibrillation and the risk of stroke," New England Journal of Medicine, vol. 366, no. 2, pp. 120-129, 2012.

[6] A.-M. Sinha, H.-C. Diener, C. A. Morillo et al., "Cryptogenic stroke and underlying atrial fibrillation (CRYSTAL AF): design and rationale," The American Heart Journal, vol. 160, no. 1, pp. 36-41, 2010.

[7] R. L. Page, W. E. Wilkinson, W. K. Clair, E. A. McCarthy, and E. L. C. Pritchett, "Asymptomatic arrhythmias in patients with symptomatic paroxysmal atrial fibrillation and paroxysmal supraventricular tachycardia," Circulation, vol. 89, no. 1, pp. 224-227, 1994.

[8] H. Kamel, M. Hegde, D. R. Johnson, B. F. Gage, and S. C. Johnston, "Cost-effectiveness of outpatient cardiac monitoring to detect atrial fibrillation after ischemic stroke," Stroke, vol. 41, no. 7, pp. 1514-1520, 2010.

[9] A. C. Flint, N. M. Banki, X. Ren, V. A. Rao, and A. S. Go, "Detection of paroxysmal atrial fibrillation by 30-day event monitoring in cryptogenic ischemic stroke: the Stroke and Monitoring for PAF in Real Time (SMART) Registry," Stroke, vol. 43, pp. 2788-2790, 2012.

[10] R. Stahrenberg, M. Weber-Krüger, J. Seegers et al., "Enhanced detection of paroxysmal atrial fibrillation by early and prolonged continuous holter monitoring in patients with cerebral ischemia presenting in sinus rhythm," Stroke, vol. 41, no. 12, pp. 2884-2888, 2010.

[11] R. C. S. Seet, P. A. Friedman, and A. A. Rabinstein, "Prolonged rhythm monitoring for the detection of occult paroxysmal atrial fibrillation in ischemic stroke of unknown cause," Circulation, vol. 124, no. 4, pp. 477-486, 2011.

[12] N. Gaillard, S. Deltour, B. Vilotijevic et al., "Detection of paroxysmal atrial fibrillation with transtelephonic EKG in TIA or stroke patients," Neurology, vol. 74, no. 21, pp. 1666-1670, 2010.

[13] P. Doliwa Sobocinski, E. Anggårdh Rooth, V. Frykman Kull, M. von Arbin, H. Wallén, and M. Rosenqvist, "Improved screening for silent atrial fibrillation after ischaemic stroke," Europace, vol. 14, pp. 1112-1116, 2012.

[14] K. Asplund, K. Hulter Åsberg, P. Appelros et al., "The RiksStroke story: building a sustainable national register for quality assessment of stroke care," International Journal of Stroke, vol. 6, no. 2, pp. 99-108, 2011.

[15] P. S. Doliwa, V. Frykman, and M. Rosenqvist, "Short-term ECG for out of hospital detection of silent atrial fibrillation episodes," Scandinavian Cardiovascular Journal, vol. 43, no. 3, pp. 163-168, 2009.

[16] H. Calkins, J. Brugada, D. L. Packer et al., "HRS/EHRA/ECAS expert consensus statement on catheter and surgical ablation of atrial fibrillation: recommendations for personnel, policy, procedures and follow-up. A report of the Heart Rhythm Society (HRS) Task Force on catheter and surgical ablation of atrial fibrillation," Europace, vol. 9, no. 6, pp. 335-379, 2007.

[17] L. Suissa, S. Lachaud, and M. H. Mahagne, "Optimal timing and duration of continuous electrocardiographic monitoring for detecting atrial fibrillation in stroke patients," Journal of 
Stroke \& Cerebrovascular Diseases, vol. 22, no. 4, pp. 991-995, 2012.

[18] C. R. Kerr and K. Humphries, "Gender-related differences in atrial fibrillation," Journal of the American College of Cardiology, vol. 46, no. 7, pp. 1307-1308, 2005.

[19] O. Alhadramy, T. J. Jeerakathil, S. R. Majumdar, E. Najjar, J. Choy, and M. Saqqur, "Prevalence and predictors of paroxysmal atrial fibrillation on holter monitor in patients with stroke or transient ischemic attack," Stroke, vol. 41, no. 11, pp. 2596-2600, 2010.

[20] J. Wohlfahrt, R. Stahrenberg, M. Weber-Krüger et al., "Clinical predictors to identify paroxysmal atrial fibrillation after ischaemic stroke," European Journal of Neurology, vol. 21, no. 1, pp. 21-27, 2013.

[21] Z. Binici, T. Intzilakis, O. W. Nielsen, L. Køber, and A. Sajadieh, "Excessive supraventricular ectopic activity and increased risk of atrial fibrillation and stroke," Circulation, vol. 121, no. 17, pp. 1904-1911, 2010.

[22] T. V. Glotzer, E. G. Daoud, D. G. Wyse et al., “The Relationship between daily atrial tachyarrhythmia burden from implantable device diagnostics and stroke risk the trends study," Circulation, vol. 2, no. 5, pp. 474-480, 2009. 


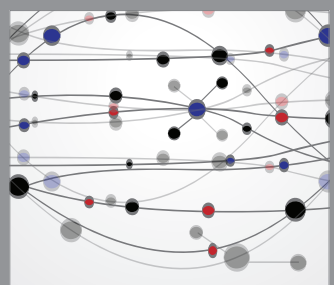

The Scientific World Journal
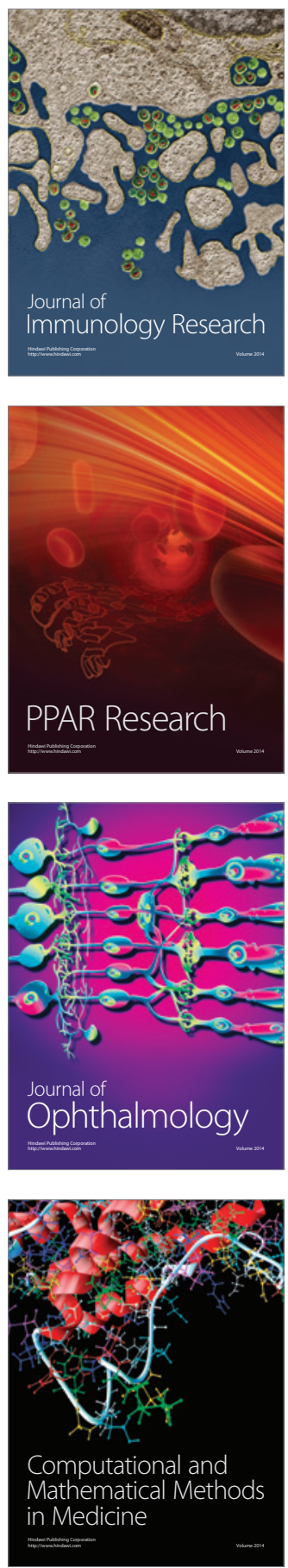

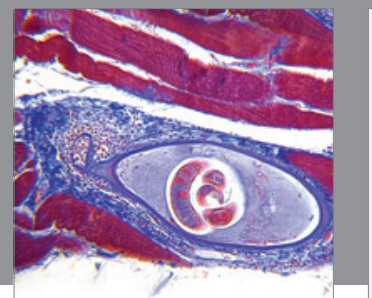

Gastroenterology

Research and Practice
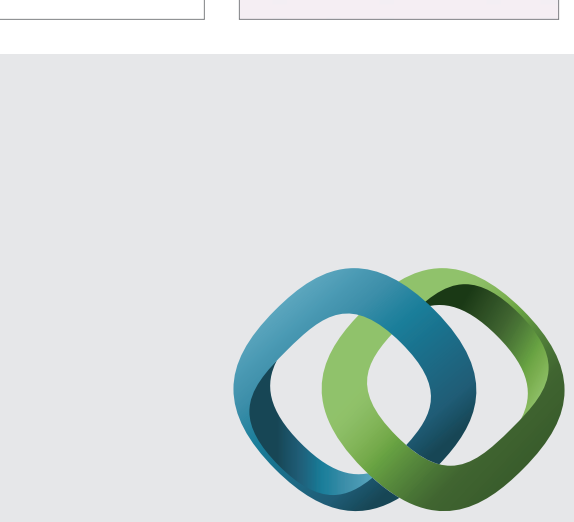

\section{Hindawi}

Submit your manuscripts at

http://www.hindawi.com
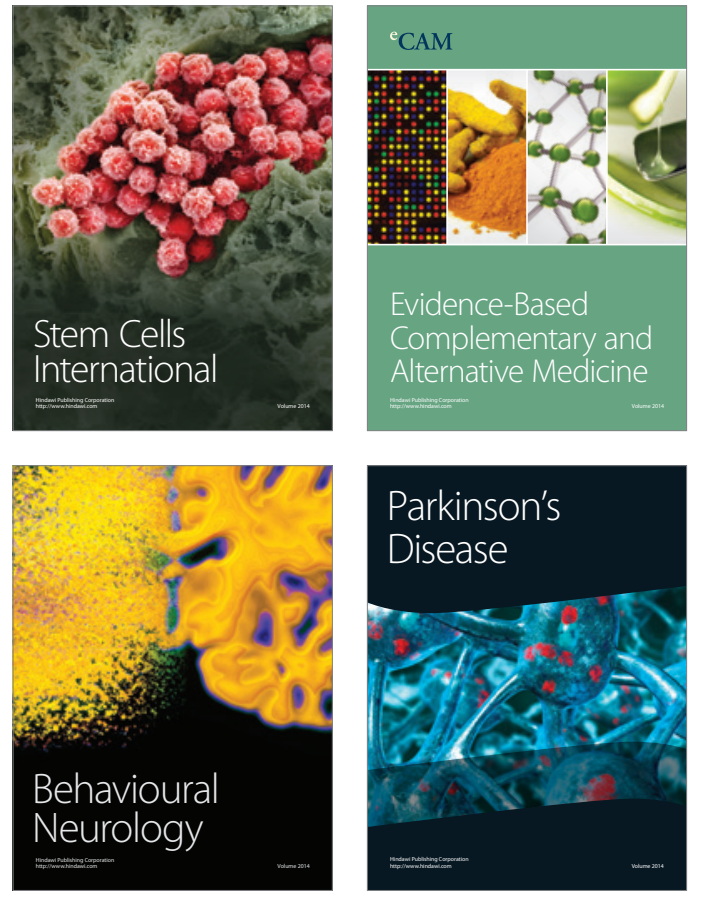
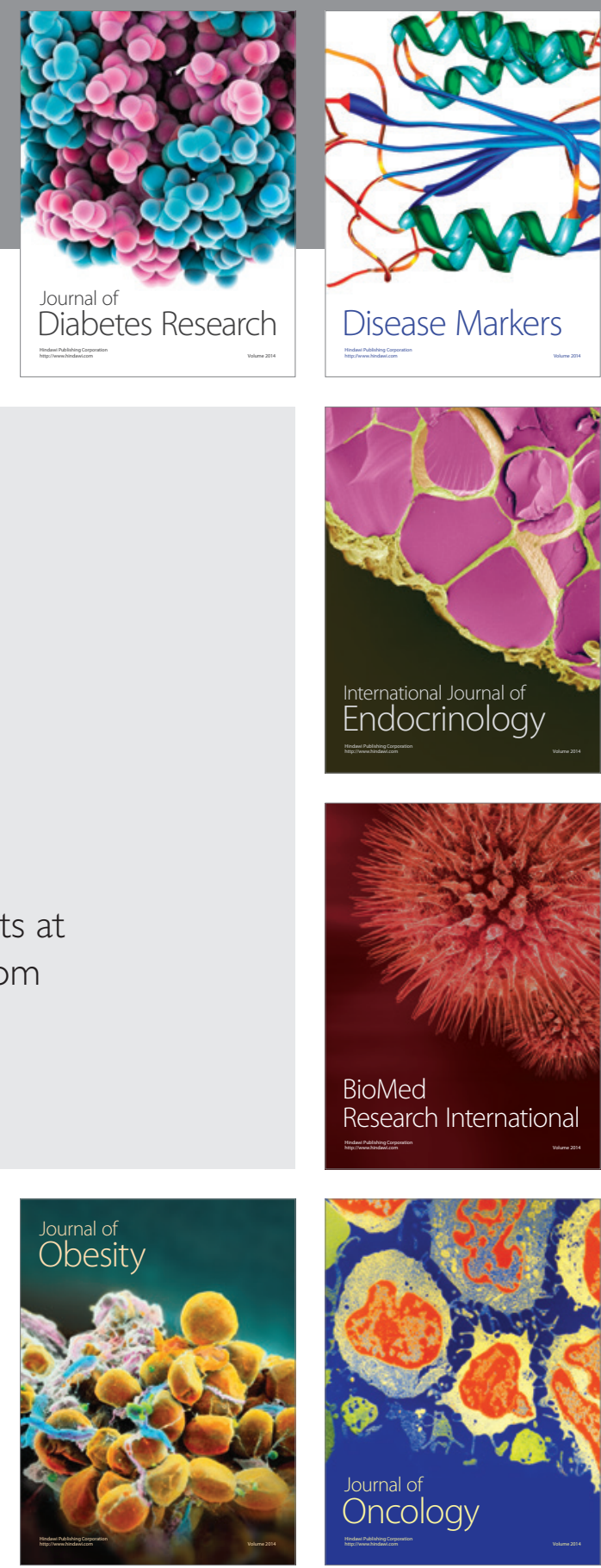

Disease Markers
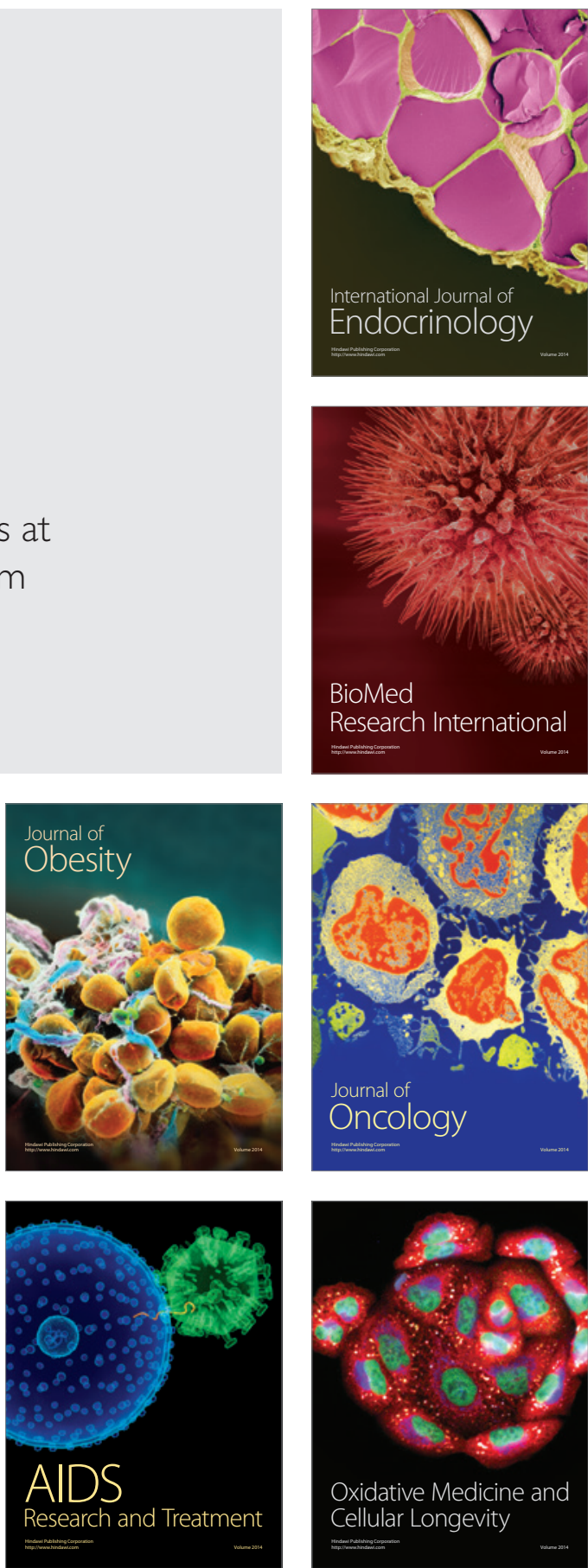